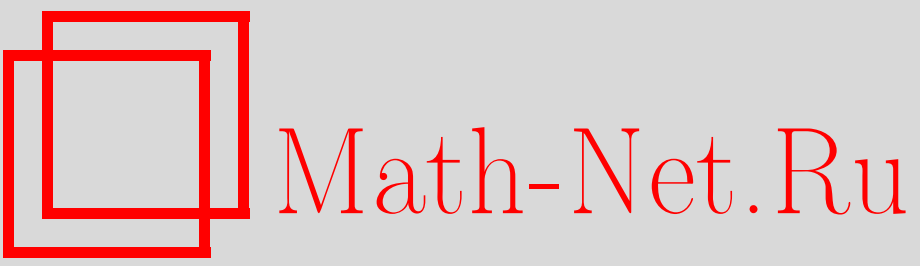

Б. М. Гуревич, Устойчиво-возвратные неотрицательные матрицы, УМН, 1996 , том 51, выпуск 3, 195-196

DOI: https://doi.org/10.4213/rm981

Использование Общероссийского математического портала Math-Net.Ru подразумевает, что вы прочитали и согласны с пользовательским соглашением

http://www.mathnet.ru/rus/agreement

Параметры загрузки:

IP : 34.239 .49 .27

26 апреля 2023 г., 12:24:16 


\title{
УСТОЙЧИВО-ВОЗВРАТНЫЕ НЕОТРИЦАТЕЛЬНЫЕ МАТРИЦЫ
}

\author{
Б. М. ГУРЕВИч
}

Конечная или беконечная квадратная матрица $Q$ с элементами $q_{i j}=q_{i j}^{(1)} \geqslant 0, i, j \in \mathbb{N}$, назьваетя неразложимой [1], если для всякой пары $(i, j)$ найдетя такое $n \geqslant 1$, что $q_{i j}^{(n)}>0\left(q_{i j}^{(n)}\right.$ - соответствующий элемент матрищы $Q^{n}$ ). Свойство неразложимости удобно выражать в геометричеких терминах. Пусть $G_{Q}$ - ориентированный граф, вершинами которого служат номера строк (и столбцов) матрицы $Q$, а ребрами - те пары $(i, j)$, для которых $q_{i j}>0$. В таком случае неразложимость $Q$ равносильна связности графа $G_{Q}$, т.е. существованию для любых двух его вершин $i$ и $j$ такого пути, который ведет из $i$ в $j$ (путем в ориентированном графе $G$, ведушим из вершины $i$ в вершину $j$, будем называть такую последовательность вершин $\gamma=\left(i_{0}, \ldots, i_{n}\right)$, что $i_{0}=i, i_{n}=j$ и $\left(i_{k}, i_{k+1}\right)$ при $0 \leqslant k \leqslant n-1-$ ребро графа $G$; будем говорить, что путь $\gamma$ содержит все свои вершины $i_{k}$ и все ребра $\left.\left(i_{k}, i_{k+1}\right)\right)$. Еще одна интерпретация этого свойства носит вероятностный характер: если $Q$ - стохастическая матрица, то она определяет цеп Маркова с конечным или счетным числом состояний, и неразложимость $Q$ означает, что состояния этой цепи образуют один существенный класс. Если эта цепь Маркова апериодична, будем также называть $Q$.

Для любого множества $V \subset \mathbb{N}$ назовем подматрицей с носителем $V$ матрицы $Q$ результат применения к $Q$ двух операций: вычеркивания всех строк и столбцов с номерами, не входящими в $V$, и замены нулями любого множества оставшихся элементов (эта терминология немного отличается от общепринятой, cp. [2]). Если упомянутой замены не происходит, подматрица назьвается главной. Любая подматрица $Q^{\prime}$ матрицы $Q$ есть снова квадратная матрица, строки и столбцы которой занумерованы числами $i \in \operatorname{supp}\left(Q^{\prime}\right)$, где $\operatorname{supp}\left(Q^{\prime}\right)-$ носитель $Q^{\prime}$. Ей (точно так же, как и $Q$ ) отвечает граф $G_{Q^{\prime}}$, являющийся подграффом графа $Q$. Очевидно, это соответствие между подматрицами и подграфами является взаимнооднозначным, причем неразложимым подматрицам отвечают связные подграфы. В дальнейшем будем писать $Q^{\prime} \subset Q$ и $G^{\prime} \subset G$, если, соответственно, $Q^{\prime}$ - подматрица матрицы $Q$ и $G^{\prime}$ - подграф граффа $G$.

Для любой неотрицательной матрицы $Q$ положим

$$
\lambda(Q)=\sup _{Q^{\prime}} \lambda\left(Q^{\prime}\right)
$$

где $\lambda\left(Q^{\prime}\right)$ - максимальное по модулю собственное число конечной подматрицы $Q^{\prime} \subset Q$ и $\sup$ берется по всем таким подматрицам (по теореме Перрона-Фробениуса $\lambda\left(Q^{\prime}\right) \geqslant 0$, см. [1]). Можно показать, что если матрица $Q$ неразложима, то в правой части (1) достаточно ограничиться конечными неразложимыми главными подматрицами $Q^{\prime}$.

Следующее определение подсказано аналогией со стохастическими матрищами.

ОпРеДЕЛЕниЕ 1 (ср. [3]). Неразложимая матрица $Q$ называетя возвратной, если при всех $i, j \in \mathbb{N}$

$$
\sum_{n} q_{i j}^{(n)}[\lambda(Q)]^{-n}=\infty
$$

и положительно-возвратной, если при всех $i, j \in \mathbb{N}$

$$
\varlimsup_{n \rightarrow \infty} q_{i j}^{(n)}[\lambda(Q)]^{-n} \neq 0
$$

(достаточно, чтобы (2) и (3) выполнялись хотя бы для одной пары $(i, j)$ ).

Основным для нас будет

Работа выполнена при частичной поддержке Российского фонда фундаментальных исследований (грант № 93-011-16-090) и Международного научного фонда (грант № M8X000). 
ОПРЕДЕЛЕНИЕ 2. Назовем неразложимую возвратную неотрицательную матрицу $Q$ устойчиво-возвратной, если для любого конечного набора $J$ пар $(i, j)$, удовлетворяюших условию $q_{i j}>0$, найдется такое $\varepsilon>0$, что, произвольно изменив $q_{i j}$ для каждой из этих пар не более чем на $\varepsilon$ и оставив неизменными остальные $q_{i j}$, мы снова получим возвратную матрицу.

Ясно, что если $\varepsilon<\min \left\{q_{i j},(i, j) \in J\right\}$, то описанное только что возмущение матрицы $Q$ не меняет графа $G_{Q}$.

Цель этой заметки - указать несколько свойств неотрицательных матриц, тесно связанных с устойчивой возвратностью, а отчасти и равносильных ей. Некоторые из этих свойств, как мы увидим, хорошо известны, другие, в частности, сама устойчивая возвратность, по-видимому, до сих пор не рассматривались. Заметим еще, что все формулируемые ниже результаты содержательны лишь для бесконечных матриц.

ТЕОРемА 1. Всякая устойчиво-возвратная неразложимая матрица положительно-возвратна; обратное неверно.

Теорема 2. Неразложимая матрица $Q$ устойчиво-возвратна в том и только том случае, когда для любой ее неразложимой собственной подматрицы $Q^{\prime}$ выполняется неравенство: $\lambda\left(Q^{\prime}\right)<\lambda(Q)$.

Для частного случая матриц, состоящих из нулей и единиц, эта теорема по существу содержится в [4].

Теорема 3. Неразложимая апериодическая стохастическая матрица $Q$ устойчиво-возвратна в том и только том случае, когда она геометрически-әргодична (см. [3]).

СЛЕДСТвиЕ. Геометрическая эргодичность неразложимой апериодической возвратной стохастической матрицы $Q$ равносильна справедливости для любой ее неразложимой собственной подматрицы $Q^{\prime}$ неравенства $\lambda\left(Q^{\prime}\right)<1$.

ЗАмечАния. 1. В формулировках теоремы 2 и следствия можно опустить требование неразложимости подматрицы $Q^{\prime}$.

2. Если бы в применении к стохастическим матрицам мы модифицировали оределение 2, потребовав, чтобы возмущенная матрица оставалась стохастической, это не привело бы ни к какому содержательному результату. С другой стороны, заметим, что в следствии фигурируют лишь сама стохастическая матрица и ее подматрицы.

Пусть $\mathscr{F}(Q)$ - совокупность неразложимых конечных главных подматриц бесконечной неотрицательной матрицы $Q$. Нетрудно показать, что если $Q$ неразложима, то $\mathscr{F}(Q) \neq \varnothing$ и, более того, найдется такая последовательность $\left\{F_{n}, n \geqslant 1\right\}, F_{n} \in \mathscr{F}(Q)$, что $F_{n} \subset F_{n+1}$ и всякий элемент матрицы $Q$ становится элементом $F_{n}$ при достаточно большом $n$. При любых $F \in \mathscr{F}(Q)$, $i, j \in \operatorname{supp}(F)$ рассмотрим множесство $\Gamma_{i j}(Q \mid F)$ путей в графе $G_{Q}$, ведуших из $i$ в $j$ и внешних по отношению к $G_{F}$, т.е. не содержаших ни одной вершины из $\operatorname{supp}(F)$, кроме $i$ и $j$. Если $i \neq j$ и $\Gamma_{i j}(Q \mid F) \neq \varnothing$, обозначим $\gamma_{j i}$ кратчайший путь в $G_{F}$, ведущий из $j$ в $i$ (любой, если их несколько). Пусть $V_{i j}(F)$ - множество вершин граффа $G_{Q}$, каждая из которых содержится в каком-нибудь пути $\gamma \in \Gamma_{i j}(Q \mid F)$ или (при $i \neq j$ ) в $\gamma_{j i}$, и $E_{i j}(F)$ - множество ребер графо $G_{Q}$, которые выделяются точно таким же условием. Введем подматрицу $Q_{i j}(F) \subset Q$, получаемую из $Q$ вычеркиванием строк и столбцов с номерами, не входящими в $V_{i j}(F)$, и заменой нулями всех $q_{m n} \mathrm{c}(m, n) \notin E_{i j}(F)$. Легко видеть, что $Q_{i j}(F)$ - неразложимая матрица (которая может быть и пустой, что будет записываться в виде $\left.Q_{i j}(F)=\varnothing\right)$.

ТЕОРема 4. Если у неразложимой матриць $Q$ найдется такая подматрица $F \in$ $\mathscr{F}(Q)$, что $\lambda(F) \geqslant \lambda\left(Q_{i j}(F)\right)$ при всех $i, j \in \operatorname{supp}(F)$, для которых $Q_{i j}(F) \neq \varnothing$, то матрица $Q$ устойчиво-возвратна.

Автор благодарен С. В. Савченко за ряд полезных замечаний.

\section{СПИСОК ЛИТЕРАТУРЫ}

[1] Гантмахер Ф. Р. Теория матриц. М.: Наука, 1967. [2] Хорн Р., Джонсон Ч. Матричный анализ. М.: Мир, 1989. [3] Vere-Jones D. // Quart. J. Math. Oxford Ser. (2). 1962. V. 13. P. 7-28. [4] Salama Ibrahim A. // Pacific J. Math. 1988. V. 134. P. 324-341.

Московский государственный университет им. М. В. Ломоносова
Принято редколлегией 15.01.1996 